\section{Estimation of General Ability}

IN statistical theory it has been for some time a discipline for statisticians to distinguish by appropriate notation a population parameter that is being estimated and the measure obtained from a sample that is to be its estimate. It is now, for example, common for a true population correlation coefficient to be denoted by $p$, and $r$ to be our sample estimate of it.

In Spearman's theory of ability there are sufficient real difficulties, without the introduction of one or two of a more artificial kind that I venture to comment on here because they seem to me to have arisen mainly owing to the non-recognition of the value of this discipline in the psychological domain. If, as a convenient notation in Spearman's two-factor theory, we denote a person's general ability by $G$, and our estimate of it by $g$, we can at once realise certain facts about $g$ that have nevertheless been put forward in rather an obscure way in the literature.

Thus $g$ will not be identical with $G$, and consequently it may be said that $G$ is indeterminate. A quantity $i$ has been introduced to represent this indeterminacy. Though, however, we may choose to write a formal equation relating $g, G$ and $i$, this will not tell us qualitatively more than we already know, that $g$ in general differs from $G$. The difference may be regarded statistically as the error of estimation; actually it must be a function of the specific abilities corresponding to the tests used. This has been pointed out recently by Prof. Godfrey Thomson ${ }^{1}$.

Further, it has been stated that $g$ is not conserved by linear transformations of the test scores. A transformation in general, since it implies that the same test score will contribute to more than one of the new test scores, will introduce group factors where none might have existed before, and correlations among the new test scores will not therefore satisfy the tetrad criterion: yet we can always regard any new value $g^{\prime}$, say, obtained from them as an estimate of $G$. We shall expect $g^{\prime}$ to differ from $g$, but this would appear to have little relevance to what value we ascribe to Spearman's theory.

Agricultural Research Station, M. S. BartLETT. Jealott's Hill, Bracknell, Berks.

${ }^{1}$ Brit. J. Psychol., 25, 92-99; 1934.

\section{Measuring General Intelligence by Tests which break the g-Hierarchy}

IN an article in the current number of the British Journal of Psychology, I have shown that two tests which fit separately into a hierarchy, but the correla. tion of which with one another breaks it, can under certain conditions be weighted so as to form a team of two tests correlating perfectly with $g$. The object of the present note is to point out an extension of this principle. If $k$ tests each fit separately into a hierarchy, but cannot co-exist in it, their correlations with $g\left(r_{1 g}, r_{2 g} \ldots r_{k g}\right)$ can be separately found. A team of these $k$ tests can then be formed, with weights proportional to (-) the co-factors of $r_{1 g}$, $r_{2 g} \ldots r_{k g}$ in the determinant

$$
\Delta=\left|\begin{array}{lllll}
1 & r_{1 g} & r_{2 g} & \cdot & r_{k g} \\
r_{1 g} & 1 & r_{12} & \cdot & r_{1 k} \\
r_{2 g} & r_{12} & 1 & \cdot & r_{2 k} \\
\cdot & \cdot & \cdot & \cdot & \cdot \\
r_{k g} & r_{1 k} & r_{2 k} & \cdot & 1
\end{array}\right|,
$$

to give an estimate of $g$, and this estimate will correlate perfectly with $g$ if the value of the above determinant is zero. If the latter is the case, the $k$ non-conforming tests may be represented as containing among them $k-1$ group components in addition to the general component $g$.

GODFREY H. THOMSON.

$$
\begin{aligned}
& \text { University, } \\
& \text { Edinburgh. } \\
& \text { Nov. 12. }
\end{aligned}
$$

\section{Dipole Moment of Iodine}

THE problem of the dipole moment of iodine is of considerable interest. Some years ago, Williams ${ }^{1}$ and Müller and Sack $^{2}$ measured the moment of iodine in benzene and carbon disulphide solutions and obtained a value of $1-1.2 \times 10^{-18}$ C.G.S. units. It is well known, however, that the iodine molecule should have a symmetrical structure. It is scarcely likely that iodine would react chemically with benzene under the conditions of their experiments. The moment observed was due to some interaction between $\mathrm{I}_{2}$ and $\mathrm{C}_{6} \mathrm{H}_{6}$. It seemed strange to us that a moment of the order of magnitude of 1 Debye, that is, a moment characteristic of a molecule of a marked polarity, should arise through the influence of Van der Waals' forces. The discrepancy between the value observed and that to be expected from theoret. ical considerations can be attributed to the imperfection of the method of measurements.

The authors mentioned above determined the dielectric constant at one temperature and calculated the moment by subtracting the electronic part, obtained from refraction data, from the total polarisation. This method is not sufficiently precise because the atomic polarisation is neglected. We have measured the dielectric constant of solutions (1-6 per cent) of iodine in benzene and carbon disulphide at different temperatures from $15^{\circ}$ to $70^{\circ}$ in the case of benzene and from $15^{\circ}$ to $35^{\circ}$ for carbon disulphide by the heterodyne beat method.

Our experiments show that iodine has no dipole moment in benzene or in carbon disulphide. The probable error of our method of investigation is not greater than $0 \cdot 1$ Debye. The electric moment of iodine is, therefore, within the limits of this error, equal to zero.

W. WASSILIEW.

J. SYRKIN.

I. KENEZ.

Laboratory of Electric Properties of Molecules, Karpow Institute for Physical Chemistry, Moscow. Nov. 28.

1 Phy8. Z., 29, 204; 1928.

2 Phys. Z., 31, 821; 1930.

\section{Designation of Logarithms to Base $e$}

IN a short review in Nature of November 3 (p. $684)$ it is remarked that "The notation 'In' for ' $\log _{e}$ ' will probably be somewhat strange to British readers".

This notation was used by Jahnke and Emde "Funktionentafeln", and even in the first (1909) edition they did not deem it necessary to explain 